Keywords: pancreatic ductal adenocarcinoma; primary cell cultures; ascites fluid; chemotherapeutic sensitivity; personalised medicine

\title{
Ascites-derived pancreatic ductal adenocarcinoma primary cell cultures as a platform for personalised medicine
}

\author{
T Golan ${ }^{1,2,5}$, D Atias ${ }^{1,5}$, I Barshack ${ }^{3}$, C Avivi ${ }^{3}$, R S Goldstein ${ }^{4}$ and R Berger ${ }^{\star, 1,2}$ \\ ${ }^{1}$ Institute of Oncology, Sheba Medical Center, Tel Hashomer 52621, Israel; ${ }^{2}$ Sackler School of Medicine, Tel Aviv University, Ramat \\ Aviv 69978, Israel; ${ }^{3}$ Department of Pathology, Sheba Medical Center, Tel Hashomer 52621, Israel and ${ }^{4}$ Mina and Everard Goodman \\ Faculty of Life Sciences, Bar-llan University, Ramat-Gan 52900, Israel
}

Background: Challenges in developing drugs for pancreatic ductal adenocarcinoma (PDAC) include obtaining metastatic cancer tissue for research and validating biomarkers predicative for personalised therapeutic decisions. We have recently developed a novel therapeutic model for PDAC to address these challenges based on the isolation of viable PDAC cells derived from ascites fluid.

Methods: Ascites fluid was obtained from PDAC patients undergoing palliative paracentesis. Ascites-derived PDAC primary cells were isolated, cultured and characterised in ovo and in vitro.

Results: We successfully established ascites-derived primary cell cultures within 2-7 days from 92\% (93 out of 101) of the ascites fluid samples obtained (from 36 different patients). Homogeneous epithelial PDAC-enriched cell cultures were identified and characterised. We observed a wide range in doubling times and migration properties among the different patient-derived cell cultures. The diverse nature of each individual patient's cell cultures was further demonstrated by differences in therapeutic susceptibility and resistance. The tumorigenicity and invasiveness of the cells were demonstrated in vivo using chicken chorioallantoic membrane grafts.

Conclusions: We have developed a unique ascites-derived PDAC primary cell culture model. This model has the potential to study signalling pathways in PDAC progression and to evaluate targeted therapies for the individual patient expeditiously, thereby supporting personalised treatment decisions.

Pancreatic ductal adenocarcinoma (PDAC) carries a dismal prognosis. Treatment options are limited with low response rates and the 5 year survival rate is below $5 \%$ when combined for all stages (Burris and Storniolo, 1997).

The discovery and development of effective cancer medicines have historically been hampered by the lack of reliable predictive preclinical models to assess the therapeutic efficacy of candidate agents. This is mostly due to the genomic heterogeneity among patients. The dependency of the cancer research community on established cell lines as models for drug development has known limitations. Therefore, alternative models that replicate this complex disease need to be developed (Feldmann et al, 2009; Sharma et al, 2010). In vivo xenograft models have partly addressed these difficulties and are being successfully used for translational drug development (Rubio-Viqueira et al, 2006; Perez-Mancera et al, 2012). However, mouse xenograft models require expertise, ethical permissions and are time and space consuming.

For solid malignancies, obtaining cancer cells at different stages of the patients' disease is challenging and often requires invasive and potentially harmful procedures. In pancreatic cancer, diagnosis

${ }^{*}$ Correspondence: Dr R Berger; E-mail: Raanan.Berger@sheba.health.gov.il
${ }^{5}$ These authors contributed equally to this work.

Received 7 August 2013; revised 9 February 2014; accepted 11 February 2014; published online 25 March 2014

(c) 2014 Cancer Research UK. All rights reserved 0007-0920/14 
is initially performed by endoscopic ultrasound fine-needle aspiration, where often times inadequate tissue sampling is obtained, limiting the availability of tissue for correlative studies. An additional source of malignant tissue is obtained from a small percentage of patients with early-stage PDAC during surgical procedures. Therefore, there is an urgent need to develop minimally invasive techniques that can acquire malignant tissue for the evaluation of candidate anticancer treatments for the patient. Approximately one-third of patients with PDAC have peritoneal spread, which may lead to ascites in the advanced disease, and palliative paracentesis is performed when clinically required (Fernandez-del Castillo et al, 1995). The ascites fluid contains viable tumour cells, and therefore it is compelling to develop investigational models based on tumour primary cells obtained from these patients' ascites.

The concept of personalised medicine consists of adapting therapeutic measures to the specificities of the patient and the biological characteristics of the tumour. Treatment outcomes are improved by targeting drugs for tumour subtypes in which they are selectively effective, with breast and lung cancers proving recent examples (Slamon et al, 2001; Lynch et al, 2004). The main challenge currently faced is to accurately define the biological parameters required for personalised medicine. Additional approaches that integrate the molecular portraits of the tumour and predict the therapeutic responses need to be developed.

We have recently developed a novel therapeutic model for pancreatic cancer to address major challenges in personalised medicine drug development, such as (i) obtaining metastatic cancer tissue for research, (ii) developing biomarkers predicative for personalised therapeutic decisions and (iii) validating these biomarkers.

\section{MATERIALS AND METHODS}

Isolation of primary pancreatic cancer cells. All ascites derived from patients were obtained following approval of the institutional ethical committee and after all patients signed an informed consent form. The conduct of the research project was approved by the Israeli Ministry of Health.

Ascites or pleural effusion was collected at the time of paracentesis for palliative reasons from pancreatic cancer patients. A range of 2-51 of collected ascites was divided into $75 \mathrm{~cm}^{2}$ tissue culture flasks, $50 \mathrm{ml}$ of ascites per flask. Flasks were incubated at $37^{\circ} \mathrm{C}$ and with $5 \% \mathrm{CO}_{2}$. To eliminate fibroblast contamination, the upper fluid was removed and placed in a fresh dish after $20 \mathrm{~min}$. The fluid was removed after 3-4 days and fresh RPMI 1640 medium supplemented with $10 \%$ FBS, $2 \mathrm{~mm}$ L-glutamine, $1 \%$ penicillin-streptomycin and $1 \%$ sodium pyruvate (Biological industries, Israel) was added and changed twice a week. Cultures were monitored for mycoplasma using the EZ-polymerase chain reaction (PCR) Mycoplasma test kit (Biological Industries).

Cell lines. The human pancreatic carcinoma cell line PANC-1 was maintained in DMEM. The human breast epithelial cell line MCF10 was maintained in a mixture of DMEM and Coon's modified F12 medium. The human melanoma cell line C8161 was maintained in RPMI 1640. All media were supplemented with $10 \%$ FBS. All the cell lines were obtained from the American Type Culture Collection (ATCC, Manassas, VA, USA).

Immunohistochemistry. $2-3 \times 10^{6}$ ascites-derived PDAC primary cells from 11 different patients were formalin fixed, dehydrated, embedded in paraffin and sectioned to $4 \mu \mathrm{m}$ slices. Similarly, masses from chick chorioallantoic membrane (CAM) grafts were formalin fixed and paraffin embedded. All immunostainings were performed on a fully calibrated Benchmark XT staining module (Ventana Medical Systems, Oro Valley, AZ, USA).
Briefly, after sections were dewaxed and rehydrated, a CC1 Standard Benchmark XT pre-treatment for antigen retrieval (Ventana Medical Systems) was performed for cytokeratin 5/6 $(\mathrm{CK} 5 / 6), \mathrm{p} 53, \alpha$-smooth muscle actin ( $\alpha$-sma) and calretinin immunostainings. Cytokeratin 7 (CK7) and cytokeratin 19 (CK19) were pre-treated with protease 1 (Ventana Medical Systems) for $4 \mathrm{~min}$. Carcinoembryonic antigen staining did not require antigen retrieval. The dilutions of the antibodies were as follows: p53 (Leica Microsystems, Newcastle, UK) 1:100, calretinin (Cell Marque, Rocklin, CA, USA) $1: 100$, CK 5/6 (DAKO, Glostrup, Denmark) 1:50, CK7 (Biogenex Laboratories, Fremont, CA, USA) 1:100, CK19 (DAKO) $1: 50$ and carcinoembryonic antigen staining (DAKO) $1: 1000$. The slides were warmed to $60{ }^{\circ} \mathrm{C}$ for $1 \mathrm{~h}$ and then processed using a fully automated protocol. Detection was performed with an UltraView detection kit and counterstained with hematoxylin (both from Ventana Medical Systems). After the immunostaining was completed, slides were dehydrated rapidly in ethanol, cleared in xylene and covered with a coverslip containing Entellan (Merck, Darmstadt, Germany).

Flow cytometry. Cells from 10 different patients were fixed in $1 \%$ paraformaldehyde for $10 \mathrm{~min}$ and permeabilised with $0.5 \%$ saponin (Sigma-Aldrich, St Louis, MO, USA). $2 \times 10^{5}$ cells were resuspended in PBS with an anti-cytokeratin-FITC antibody, anti-CD44-FITC antibody and anti-CD90-vio770 antibody. Cells were analysed on a FACSCalibur using Winmdi software (winmdi.software.informer.com). All antibodies were from Miltenyi Biotec (San Diego, CA, USA).

Migration and invasion assays. $1 \times 10^{5}$ cells per well were seeded in triplicate on Transwell ThinCerts PET $8-\mu \mathrm{m}$ membranes (Greiner-Bio-One, Frickenhausen, Germany) with 0.5\% FBS media, placed in 24-well plates with $10 \%$ FBS media and cultured for $24 \mathrm{~h}$. The upper well content was removed using cotton swabs and the number of cells that migrated was estimated using an XTT cell proliferation kit (Biological Industries) according to manufacturer's instructions. Cell numbers were normalised to OD values of known numbers of cells. For the invasion assays, $3 \times 10^{4}$ cells per well were seeded in Matrigel (BD Biosciences, San Jose, CA, USA) pre-coated inserts. C8161 cells (a highly invasive cell line) were used as a positive control.

In-ovo. Fertile chicken (Gallus gallus) eggs were obtained from a local farm. On embryonic day 8 , a window was made in the shell exposing the CAM. $1-3 \times 10^{6}$ ascites fluid-derived PDAC primary cells mixed with Matrigel (BD Biosciences) in a total volume of $100 \mu \mathrm{l}$ were transplanted onto the CAM onto a $1-\mathrm{cm}$-diameter polypropylene ring, the eggs were then sealed and incubated for an additional 8 days. Chicken embryonic liver tissues were dissected for PCR and histological analysis. Matrigel alone and Matrigel with MCF10 cells (a non-tumorigenic epithelial cell line) served as controls.

PCR analysis. DNA was extracted from chicken embryo's liver using a DNeasy Blood \& Tissue kit (Qiagen, Hilden, Germany). Human DNA was detected using primers to the human-specific alpha-satellite sequences (Grinberg et al, 2009). The number of cycles was 35 . Human DNA isolated from patient cells (positive control) and chicken DNA (negative control) was run with each PCR reaction. The alpha-satellite amplification product is $476 \mathrm{bp}$. The primers were as follows: forward, $5^{\prime}$-GGGATAATTTCAGCT GACTAAACA- $3^{\prime}$ and reverse, 5'-AAACGTCCACTTGCAGAT TCTAG-3' (Grinberg et al, 2009).

K-RAS mutational status analysis. DNA was isolated from ascites-derived PDAC cultures using DNeasy Blood and Tissue kit (Qiagen), followed by quantification in a NanoDrop spectrophotometer (Thermo Scientific, Pittsburgh, PA, USA). K-RAS exon 2 was amplified by PCR from $100 \mathrm{ng}$ genomic DNA using PCR 
ReddyMix (Thermo Scientific). The primers were as follows: forward, $5^{\prime}$-TCATTATTTTTATTATAAGGCCTGCTGAA- $3^{\prime}$ and reverse, $5{ }^{\prime}$-CAAGATTTACCTCTATTGTTGGATCATATTC- $3^{\prime}$. The PCR reaction consisted of $5 \mathrm{~min}$ at $96^{\circ} \mathrm{C}$, followed by 35 cycles at $94{ }^{\circ} \mathrm{C}$ for $30 \mathrm{~s}, 58^{\circ} \mathrm{C}$ for $45 \mathrm{~s}$ and $72^{\circ} \mathrm{C}$ for $45 \mathrm{~s}$, and finally at $72{ }^{\circ} \mathrm{C}$ for $10 \mathrm{~min}$. PCR product was purified using Wizard SV Gel and PCR Clean-Up System (Promega, Fitchburg, WI, USA). $K-R A S$ exon 2 was sequenced using sequencing service (HY Labs, Rehovot, Israel) using the new ABI 3730xl DNA Analyzer. The sequencing results were observed by Chromas2 software (chromas.software.informer.com/2.4/) and compared with the reference sequence of $K-R A S$ gene from NCBI database to mark the position of nucleotide change.

Real-time PCR analysis. RNA was isolated using TRIZOL reagent (Invitrogen, Life Technologies, Grand Island, NY, USA) and was used as a template in the reverse transcription reaction using the ReadyMix PCR Master Mix (Thermo Scientific) according to the manufacturer's protocol. QRT-PCR was performed using SYBRGreen Master Mix (Applied-Biosystems, Life Technologies) on a 7500 Real-Time PCR system (Applied-Biosystems). Gene expression was normalised to GAPDH. Primer sequences are listed in the supplementary data (Supplementary Table S1). All primers were purchased from Sigma-Aldrich.

Chemotherapeutic assays. $2 \times 10^{3}$ ascites-derived PDAC primary cells per well from 14 different patients were seeded in 96-well plates and cultured for 3-4 days. The cells were treated with different chemotherapeutic agents: gemcitabine $10 \mu \mathrm{M}$ (Medac, Wedel, Germany), erlotinib $10 \mu \mathrm{M}$ (Roche, Basel, Switzerland), cisplatin $1 \mu \mathrm{M}$ (Pharma-Chem, Haarlem, Holland), 5FU $5 \mu \mathrm{M}$ and oxaliplatin $6 \mu \mathrm{M}$ (Ebewe, Unterach, Austria), irinotecan $10 \mu \mathrm{M}$ (Hospira, Melbourne, Australia) and paclitaxel $1 \mu \mathrm{M}$ (Teva, Petach Tikva, Israel) for $72 \mathrm{~h}$. FOLFIRINOX is a combination of 5FU, irinotecan and oxaliplatin. Cell proliferation was measured using the XTT cell proliferation kit (Biological Industries) according to the manufacturer's protocol.

Patients' clinical characteristics. Data on patient demographics, clinical history, surgery, systemic treatment, and responses to treatment and germ-line mutation of BRCA 1/2 were collected from the Sheba Medical Center cancer database and patient records. Clinical stages were classified according to the TNM AJCC staging system after review of the patients' medical, operative and pathology reports. Responses to treatment were categorised according to imaging results.

Statistical methods. The reported values represent the means \pm s.d. for experiments performed in triplicates. The significance of differences between experimental variables was determined using Student's $t$-test.

\section{RESULTS}

PDAC primary cell culture. To culture the ascites-derived PDAC primary cells, we utilised a protocol based on a rapid and simple method described by Shepherd et al (2006) with the following modifications: seeding the ascites fluid with no supplements and removal of the medium to eliminate fibroblast contamination 20 min after seeding the cells. We successfully established ascitesderived primary cell cultures within 2-7 days in 92\% (93 out of 101) of cases. In a period of 24 months, a total of 93 successful cultures were established from 36 different patients.

Cells adhered to the tissue culture dish as monolayer. The erythrocytes and lymphocytes were removed with the medium change after 3-4 days. Subsequently, cells were grown to $85-90 \%$ confluency and experiments were conducted during passages 1-3.
Cells entered senescence after 5-6 passages in the majority of the ascites cell cultures.

Morphological characterisation of PDAC primary cells. The most common morphology of the ascites-derived PDAC primary cell cultures was a cobblestone monolayer, characteristic of epithelial cells (Figure 1A). In 20 patients we obtained additional cultures during the progression of their disease (ranging from weeks to months); in 3 of these patients we observed morphological changes in the cultures between the initial and late harvesting. These changes included elongated cells with a spindle-like appearance and the loss of epithelial morphology characteristics (Figure 1B).

To further characterise the ascites-derived cells and to confirm their PDAC origin, IHC staining was performed on cells from 11 different patients. All the samples analysed clearly showed positive staining of epithelial marker CK7. Samples were negative to cytokeratin 5/6 excluding the mesothelial origin of the cells (Chu and Weiss, 2002). Additional positive staining for CK 19 and CA 19-9 was observed in some of the samples examined.

We further compared IHC markers of PDAC tissue obtained from a patient's curative resection to the same patient's ascitesderived cells obtained several months later (Figure 1C). Similar staining between the two specimens is shown for CK 7 and CK 5/6. Cytokeratin staining by FACS was observed in $90-95 \%$ of the isolated cells from different cultures including cultures displaying spindle-like morphology (Figure 1D).

To exclude stromal contamination, we demonstrated low staining to $\alpha$-sma stromal marker (IHC) and CD90 (FACS) of the isolated cells from different cultures including cultures displaying spindle-like morphology (Figure 1E; Kisselbach et al, 2009; Neesse et al, 2011).

Taking together the IHC and the FACS data, these experiments demonstrate the epithelial origin of the ascites-derived cells isolated from advanced pancreatic cancer patients.

K-RAS status. K-RAS status was analysed in cells from ascitesderived PDAC primary cells obtained from 16 different patients. 14 samples showed WT K-RAS and 2 samples showed K-RAS mutation (mutG12R). Four of these 16 samples had accompanying primary tumour from resection. K-RAS mutation (mutG12R) was detected in tissue from one patient's primary resected and KRAS WT in the other three patients' primary samples. Accordance of K-RAS status was observed between primary tumour and ascites cells.

Functional characterisation of the ascites-derived cells. To study functional characteristics of the ascites-isolated cells from PDAC patients, we compared the doubling time between cultures from eight different patients and an established cell line (PANC-1). The patients' cultures demonstrated varying proliferation rates (Figure 2A). In all of the cultures the rate was lower (range 7-14 days) when compared with PANC-1 (1.5 days).

We then analysed the migration and invasion properties of the different patients' ascites-derived PDAC cell cultures and compared them with PANC-1. Most of the cell cultures analysed displayed higher migratory rates (eight different cultures) and more invasive characteristics (six different cultures) compared with the PANC-1 (Figure 2B and C). Interestingly, we also observed an increase in the migration and invasion properties in the same patient (patient 17) during the progression of the disease.

Cells displaying epithelial-mesenchymal transition characteristics. As was described above, in some patients we cultured ascites-derived PDAC primary cells at different time points of their disease. The cultures displayed morphological changes as the disease progressed. In most cases, the cells initially displayed a characteristic epithelial morphology. Although demonstrating epithelial phenotypes, the cells were exclusively negative to 

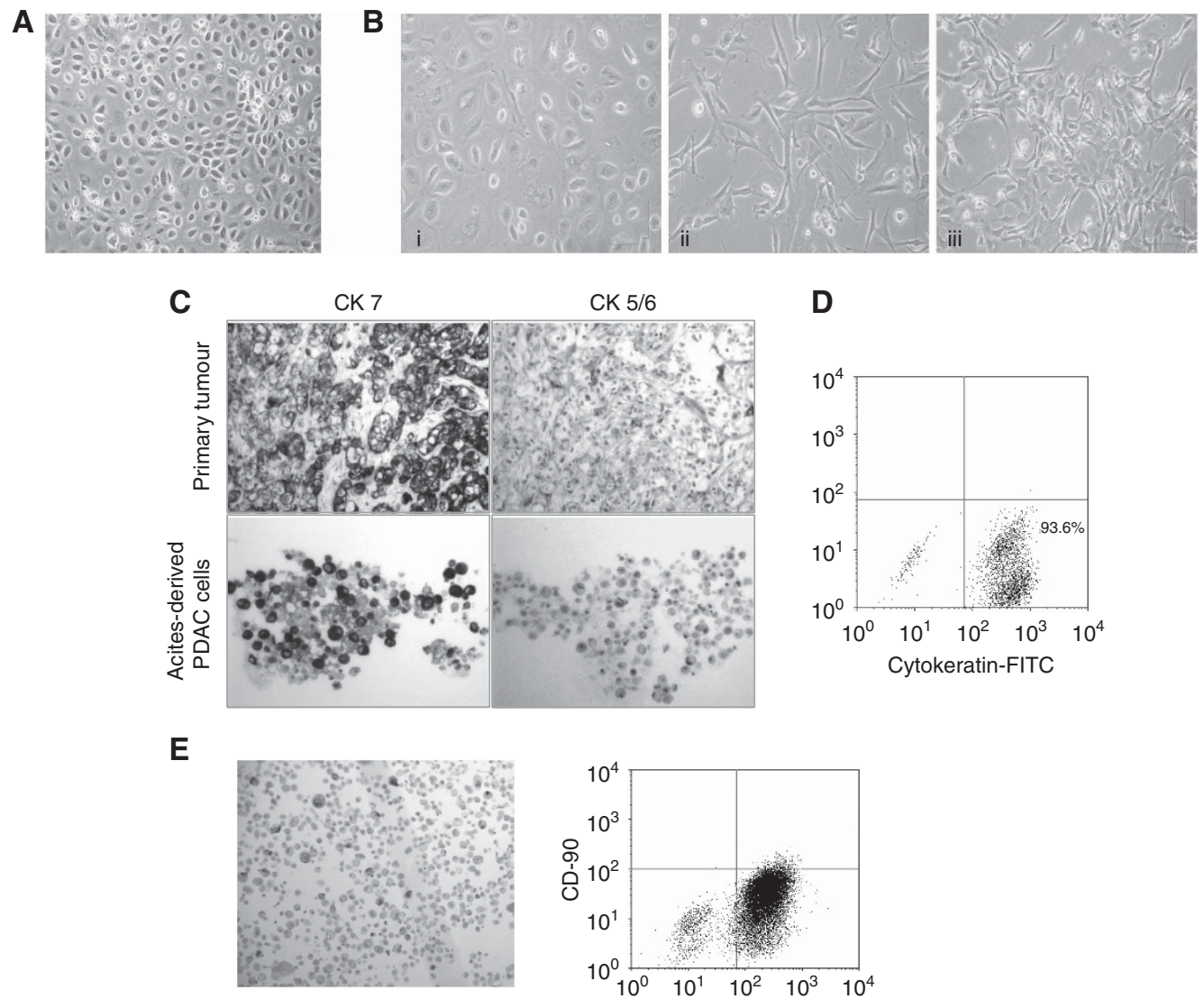

$\alpha$-SMA

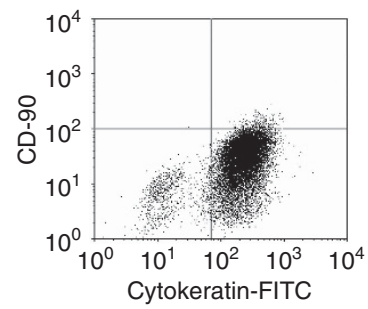

Figure 1. Morphology and identification of ascites-derived PDAC primary cells. (A) A confluent monolayer depicting a typical epithelial morphology with a polygonal shape and tight cell-to-cell junction. Scale bars, $100 \mu \mathrm{m}$. (B) Ascites-derived PDAC primary cultures from the same patient at different periods of the disease. (i) Cultures from initial ascites obtained from patient who displayed a characteristic epithelial morphology. (ii) Cultures from ascites obtained 4 weeks later displayed a mixed morphology of 'epithelial-mesenchymal'. Two predominant cell populations were observed, characteristic epithelial cells and spindle-like cells. (iii) Cultures from ascites obtained 8 weeks later - cells display predominately a spindle-like appearance. Scale bars, $100 \mu \mathrm{m}$. (C) Comparison of immunostaining of epithelial (CK7) and epithelioid mesotheliomas (CK 5/6) markers. In the upper panel: PDAC tissue obtained from patient's primary tumour during curative resection and in the lower panel: ascitesderived PDAC cells obtained from the same patient several months later. Similar staining between the two specimens is demonstrated. (D) FACS staining of epithelial/epithelial origin marker pan-cytokeratin-FITC supporting the presence of homogeneous epithelial PDAC-enriched cell culture. (E) Low IHC and FACS staining of stromal markers, $\alpha$-SMA and CD90 in ascites-derived PDAC cells.
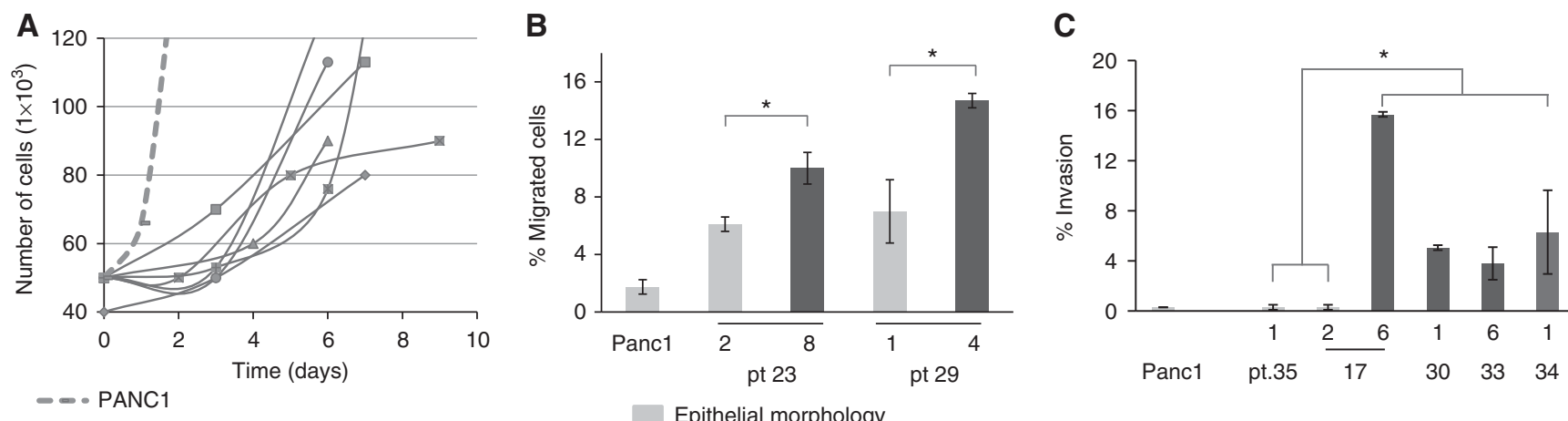

Epithelial morphology

Figure 2. Ascites-derived PDAC primary cultures' functional characteristics. (A) Growth curve of PDAC primary cells from eight different patients. (B) Varying migration properties in two different patients during the progression of their disease is shown. A significant increase in migration activity was observed during diseases progression. Values represent the means \pm s.d. $\left({ }^{\star} P<0.05\right)$. (C) Varying invasive properties in six different cultures from five different patients is depicted. In cell cultures displaying spindle-like morphology, a significantly higher fraction of cells with invasion properties was observed. Values represent the means \pm s.d. $\left({ }^{\star} P<0.05\right)$. 
A

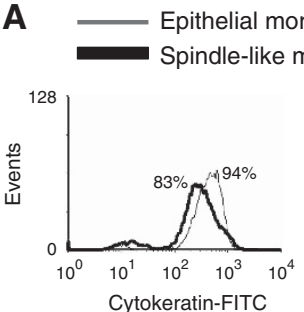

B

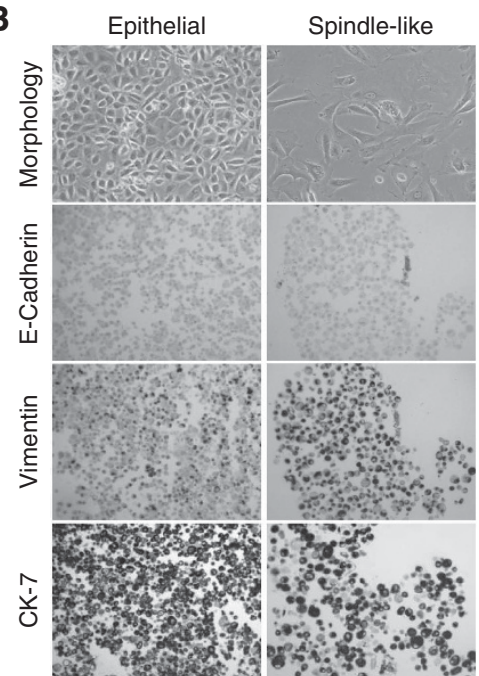

C

Epithelial morphology

Spindle-like morphology
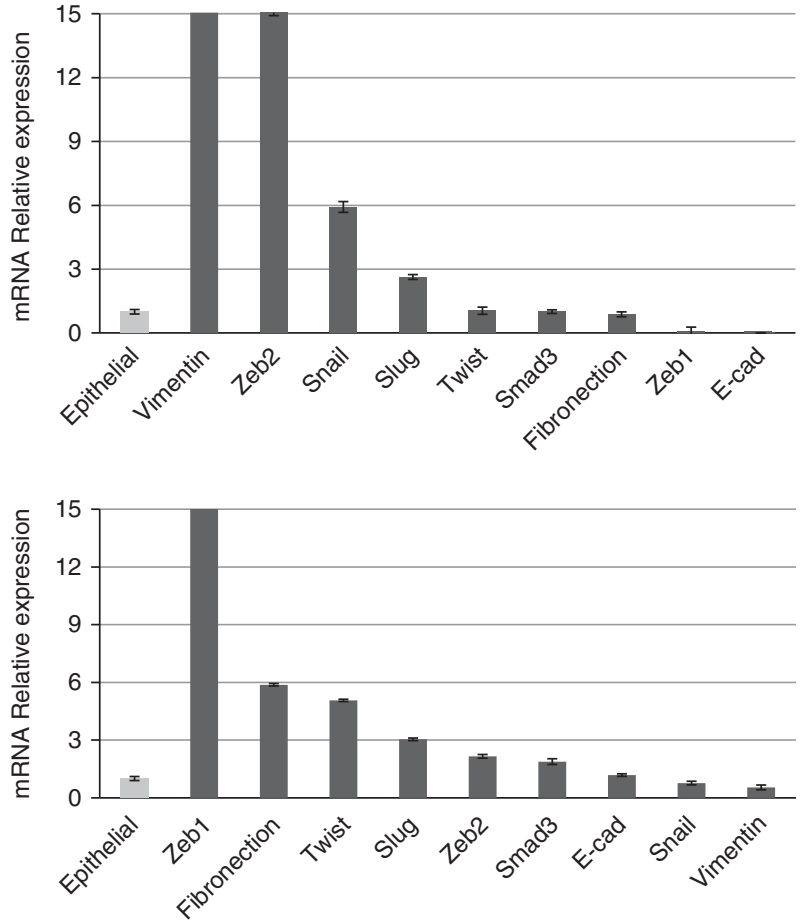

Figure 3. Overexpression of EMT characteristics in cells displaying a spindle-like morphological appearance. (A) A similar cytokeratin FACS staining between epithelial morphology cells and the spindle-like morphology cells. However, a significant higher fraction of $\mathrm{CD}_{4}{ }^{+}{ }^{+}$cells was observed in cells displaying spindle-like morphology. (B) Cells displaying epithelial vs spindle-like morphology and their representative IHC staining: E-cadherin, vimentin and CK7. (C) Real-time PCR EMT markers' expression: comparison of cultures with epithelial vs 'spindle-like' morphology in two patients. Several EMT markers are upregulated in the cultures displaying 'epithelial-mesenchymal' morphology. All differences are significant. In each case, values represent the means \pm s.d. $(P<0.05$, compared with epithelial morphology as control).

E-cadherin. Spindle cell morphology appeared with the advancement of the disease. Vimentin was overexpressed selectively in the cultures characterised with spindle cell morphology (Figure 3B). We performed comparative experiments in these four patients during the progression of their disease. Our data demonstrated that cells displaying spindle cell morphology had similar FACS cytokeratin staining (90-95\%), thus eliminating fibroblast overgrowth. In addition, we observed a higher cancer stem cell marker, CD44 (Figure 3A), and upregulation of epithelial-mesenchymal transition (EMT) markers, including Zeb-1, Zeb-2, Twist, Slug and Dab2 (Figure 3C), compared with cells with epithelial cobblestone appearance. These results suggest that spindle cells' morphology in ascites-derived cells have a more advanced EMT process, suggesting further disease invasiveness and progression.

In vivo characterisation of ascites-derived PDAC cells. To study the tumorigenicity of the ascites-derived PDAC primary cells, we utilised the model system of transplanting cells to the CAM of fertilised chicken eggs. The number of eggs xenografted was determined by the number of primary cancer cell available obtained from eight different patients $(n=25)$. A mass was detected in the ring on the CAM in 4 out of 4 MCF10 celltransplanted embryos and in 3 out of 6 Matrigel-only transplanted embryos (diameters $0-2.8 \mathrm{~mm}$ ). Somewhat larger masses were detected on the CAM of 23 out of 25 ascites-derived PDAC primary cell-transplanted embryos (diameters $1-6 \mathrm{~mm}$ ). In one graft derived from PDAC cells, a mass with invading blood vessels from the host was observed (Figure 4A). Engraftment of ascitesderived PDAC primary cells on the CAM mass was demonstrated by H\&E and IHC staining of the human cancer cell marker CK7.
Strong positive CK7 staining was detected in 11 out of 11 samples examined. In H\&E staining, we observed chick (nucleated) erythrocytes and immune system cells in all grafts. However, human cells were only observed in the MCF10 and ascites-derived PDAC primary cell-transplanted embryos. Furthermore, human DNA was detected in liver tissue of ascites-derived PDAC primary cells' CAM-transplanted embryos from one patient (pt.35) but not in the liver from pt.38 engrafted embryos or MCF10 cells control. These results once again show the diversity in behaviour of ascitesderived PDAC primary cells from different patients (Figure 4B).

Personalised therapeutic results. Our ultimate goal is to utilise this model system for personalised treatment for PDAC patients in a short time frame. Therefore, we initially tested drug therapeutic sensitivity by measuring the cells' proliferative response to different chemotherapies and biological agents (Figure 5). Importantly, we were able to finalise therapeutic results from initial harvesting of cells in a range of 7-10 days. Our results demonstrate the diverse nature of each individual patient's cells' varying therapeutic susceptibility to gemcitabine and for erlotinib (inhibitor of epidermal growth factor receptor) (Figure 5A). Varying therapeutic susceptibility to other chemotherapies was observed (data not shown).

The following examples demonstrate correlation between the in vitro results and the correspondent clinical outcomes:

Patient 36: a 78-year-old female presented with malignant ascites at diagnosis and resistance to first-line gemcitabine. Her corresponding pre-treatment ascites cells demonstrated initial in vitro gemcitabine resistance ( $86 \%$ cell viability). Ascites collected after treatment displayed a significantly higher drug resistance (110\% cell viability) (Figure 5B). 


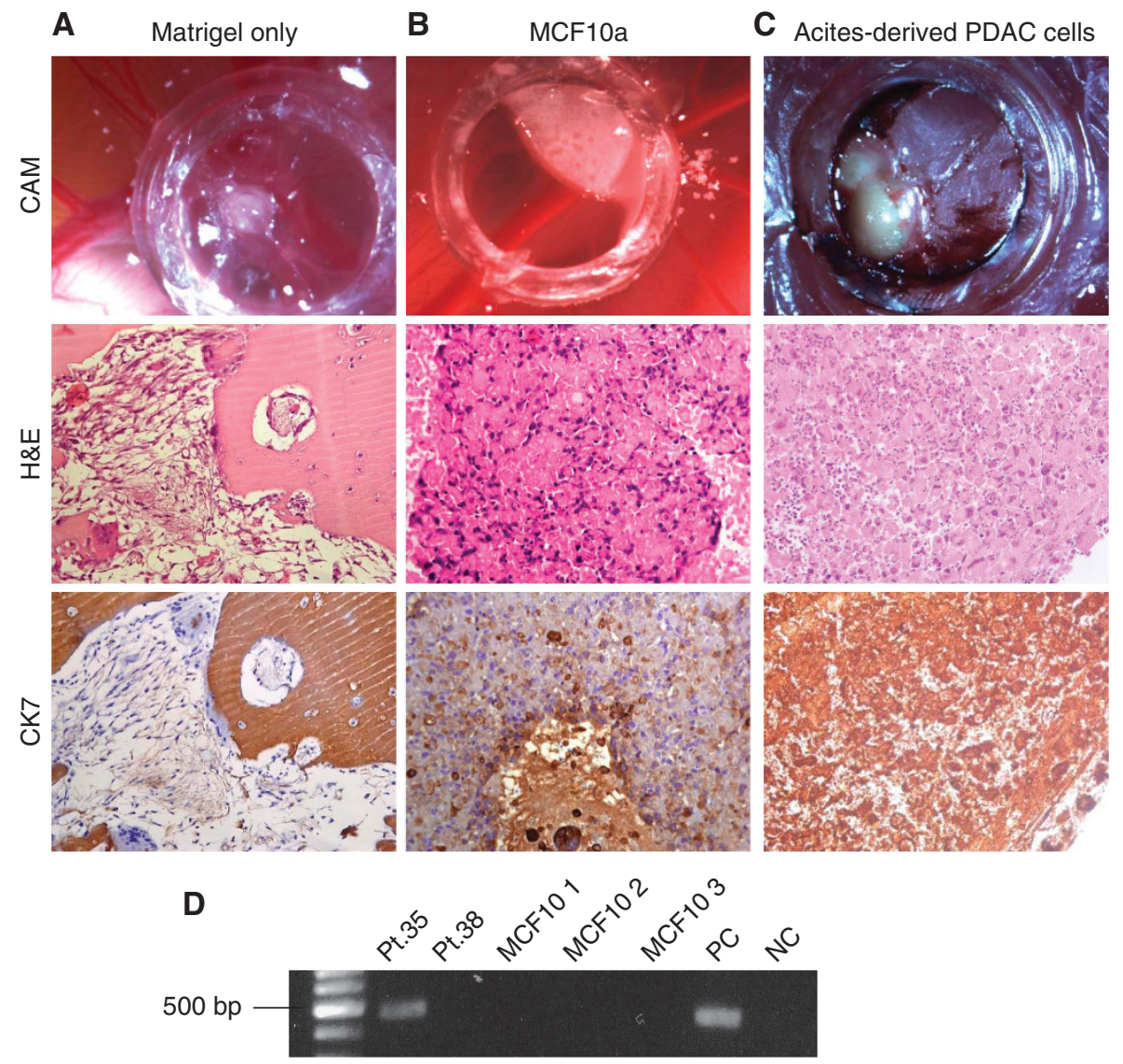

Figure 4. Ascites-derived PDAC primary cells in ovo. Cells transplanted in a ring on the CAM. (A) Grafts of Matrigel-only result in a Matrigel mass containing invading avian cells and that does not stain for human CK7. (B) MCF10 cells engraft and form a mass with weak CK7 staining. (C) Ascites-derived PDAC primary cells form a mass that is CK7 positive. (D) Detection of human alpha-satellite DNA in DNA isolated from livers of embryos transplanted with cells derived from patient 35 (pt.35), pt.38 and MCF10 cells. Human DNA was detected in one pt.35) sample. DNA extracted from cultured cells was used as a positive control (PC).
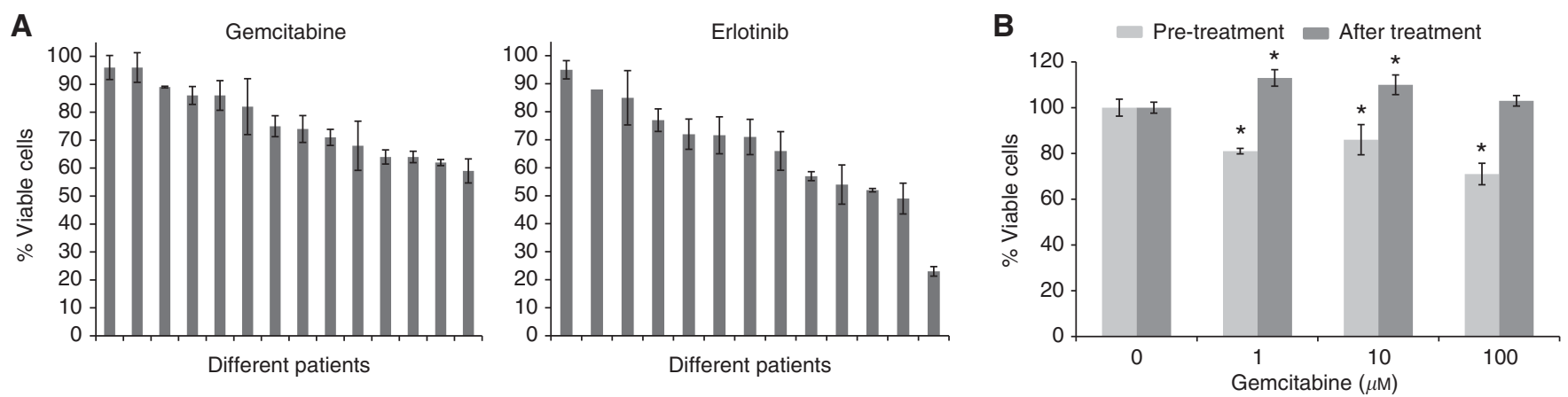

Figure 5. Ascites-derived PDAC primary cultures display varying chemotherapeutic responses. (A) Varied responses to gemcitabine $10 \mu \mathrm{M}$ and erlotinib $10 \mu \mathrm{m}$ in cells obtained from 14 different patients. (B) Two different cell cultures from the same patient before and after the patient was treated with gemcitabine. In each case, values represent the means \pm s.d. ( ${ }^{\star} P<0.05$, compared with control). All concentrations are in $\mu \mathrm{m}$. Viability of cells was measured using the XTT cell proliferation kit.

Patient 37: a 55-year-old male with liver metastasis showed a good clinical response to first-line treatment with gemcitabine and erlotinib (duration of response was 8 months). Ascites obtained during first-line treatment demonstrated in vitro sensitivity to gemcitabine alone ( $79 \%$ cell viability), erlotinib alone $(85 \%$ cell viability) and to the combination ( $68 \%$ cell viability) in correlation with the clinical response.

Patient 48: a 60-year-old female initially diagnosed with locally advanced inoperable pancreatic cancer. Ascites obtained before initiation of second-line treatment with FOLFOX demonstrated susceptibility to oxaliplatin ( $46 \%$ cell viability), in correlation with the patients good clinical response to FOLFOX (radiological partial response and progression-free survival of 6 months).

Patient 47: a 67-year-old BRCA 2-mutation carrier demonstrated a short but marked response to gemcitabine and cisplatin with a significant improvement in pain control and a $90 \%$ reduction in tumour marker CA 19-9. Pre-treatment of ascites showed in vitro sensitivity to gemcitabine and cisplatin $(63 \%$ cell viability) and post-treatment ascites obtained at tumour progression demonstrated resistance (86\% cell viability). 
These results reflect the correlation between the in vitro results and the clinical outcomes and further suggest the reliability of our personalised approach.

Our model system is based on the linkage and correlation between the ascites-derived PDAC cells and the clinical course of the correspondent patient. We have summarised the clinical characteristics of 33 patients that we successfully established primary cells cultures from the fluid obtained at palliative paracentesis/pleurocentesis (Supplementary Data, Supplementary Table 2). The clinical characteristics include: demographics, stage at diagnosis, metastatic sites, types and duration of treatments and germ-line mutation carrier status.

\section{DISCUSSION}

We have isolated and characterised PDAC cells utilising an expeditious method as a platform for personalised approach to treat PDAC patients. Using this method we have established 93 cultures from 36 patients at different time points. This method allows the investigation of PDAC in both different patient and for the same patient throughout the progression of the disease. This method can help us in our understanding of this lethal disease and it gives us a source of cancer cells where normally the availability of PDAC tissue is scant.

We further identified a homogeneous PDAC cell population in the cultures. This finding is supported by the IHC staining of CK 7 and FACS positive for cytokeratin in above $90 \%$ of the cells. Although the cultures displayed PDAC cells predominantly, the isolated PDAC cells from each of the patients displayed varying functional characteristics including different doubling times, migration, invasion properties and responses to therapeutic agents. The diversity observed in all parameters measured on the isolated PDAC cells from different patients reflects the genomic heterogeneity of pancreatic cancer.

Surprisingly, the majority of our samples analysed demonstrated WT KRAS status. Our results suggest predominance for peritoneal spread and ascites accumulation in the KRAS WT subpopulation in PDAC. The PDAC-malignant ascites fluid is understudied and we intend to further study the mechanisms underlying this observation.

The morphological and functional changes observed in PDAC cells obtained from patients during the progression of their disease led us to investigate the EMT phenomena. Our data show that most of the isolated ascitic cells were negative to E-cadherin, suggesting that all of the cells were in the spectrum of the EMT transition (Polyak and Weinberg, 2009). Furthermore, vimentin was overexpressed selectively in the cultures characterised with spindle cell morphology. In addition, these cells show upregulation of EMT genes and a higher invasion capacity in the cells with spindle cell morphological changes. These findings warrant further investigation of EMT in pancreatic cancer together with additional relevant pathways.

To our knowledge this is the first time ascites-derived PDAC cells from different patients have been grafted to chick CAM, although the chick CAM is well established as an in vivo model for studying transformed human cells (Hagedorn et al, 2005; Grinberg et al, 2009). The establishment of cell masses on the CAM is consistent with in vitro studies of tumorigenicity of the PDAC primary cell cultures. We are currently further investigating this in vivo model for drug testing and development with different chemotherapeutic agents.

Cultured cancer cells are the most widely used resource for drug sensitivity assays. Two recently published articles have addressed therapeutic sensitivity in correlation to molecular basis from hundreds of cell lines including 17 and 41 established pancreatic cell lines (Barretina et al, 2012; Garnett et al, 2012). The information obtained from the two data sets can be potentially integrated with molecular and pharmacological profiles obtained from additional panels of cell cultures.

We are currently going a step further and we are utilising primary pancreatic cells. The goal is to find a correlation between molecular profiling and therapeutic sensitivity for each individual patient. Using ascites is limited to the sampling of PDAC cells from patients with ascites/pleural effusion and not from all PDAC patients, this is the disadvantage of our model. There may also be genomic and biological differences in the ascites patient population. Therefore, our results may not be applicable to all PDAC patients.

The ascites-derived pancreatic cancer primary cell culture model has, however, several advantages in comparison with the currently available models. These benefits include: (1) the retrieval of abundant PDAC cells from patients with advanced disease as part of a standard medical care procedure. (2) The collection of cancer cells from patients at an advanced stage of their disease allows the further investigation of relevant signalling pathways in PDAC. (3) The establishment of PDAC cell cultures from different patients replicates the diversity of the genomic heterogeneity of PDAC. (4) The susceptibility to a therapeutic agent can be assessed on each individual culture in a short time frame and ultimately allows personalised treatment decisions.

In summary, we have developed a unique ascites-derived PDAC primary cell culture model. This model has the potential to allow the study of signalling pathways in PDAC progression and to evaluate targeted therapies for the individual patient expeditiously, thereby supporting personalised treatment decisions.

\section{ACKNOWLEDGEMENTS}

We are indebted to the patients who donated their cells and time; to the clinical teams who facilitated sample acquisition; and to Drs Keren Levanon, Shoshana Paglin and Keren Bachar for their guidance in initial harvesting technique.

\section{CONFLICT OF INTEREST}

The authors declare no conflict of interest.

\section{REFERENCES}

Barretina J, Caponigro G, Stransky N, Venkatesan K, Margolin AA, Kim S, Wilson CJ, Lehar J, Kryukov GV, Sonkin D, Reddy A, Liu M, Murray L, Berger MF, Monahan JE, Morais P, Meltzer J, Korejwa A, Jane-Valbuena J, Mapa FA, Thibault J, Bric-Furlong E, Raman P, Shipway A, Engels IH, Cheng J, Yu GK, Yu J, Aspesi Jr. P, De Silva M, Jagtap K, Jones MD, Wang L, Hatton C, Palescandolo E, Gupta S, Mahan S, Sougnez C, Onofrio RC, Liefeld T, Macconaill L, Winckler W, Reich M, Li N, Mesirov JP, Gabriel SB, Getz G, Ardlie K, Chan V, Myer VE, Weber BL, Porter J, Warmuth M, Finan P, Harris JL, Meyerson M, Golub TR, Morrissey MP, Sellers WR, Schlegel R, Garraway LA (2012) The Cancer Cell Line Encyclopedia enables predictive modelling of anticancer drug sensitivity. Nature 483: 603-607.

Burris H, Storniolo AM (1997) Assessing clinical benefit in the treatment of pancreas cancer: gemcitabine compared to 5-fluorouracil. Eur J Cancer 33(Suppl 1): S18-S22.

Chu PG, Weiss LM (2002) Expression of cytokeratin 5/6 in epithelial neoplasms: an immunohistochemical study of 509 cases. Mod Pathol 15: 6-10.

Feldmann G, Rauenzahn S, Maitra A (2009) In vitro models of pancreatic cancer for translational oncology research. Expert Opin Drug Discov 4: 429-443.

Fernandez-del Castillo C, Rattner DW, Warshaw AL (1995) Further experience with laparoscopy and peritoneal cytology in the staging of pancreatic cancer. Br J Surg 82: 1127-1129. 
Garnett MJ, Edelman EJ, Heidorn SJ, Greenman CD, Dastur A, Lau KW, Greninger P, Thompson IR, Luo X, Soares J, Liu Q, Iorio F, Surdez D, Chen L, Milano RJ, Bignell GR, Tam AT, Davies H, Stevenson JA, Barthorpe S, Lutz SR, Kogera F, Lawrence K, Mclaren-Douglas A, Mitropoulos X, Mironenko T, Thi H, Richardson L, Zhou W, Jewitt F, Zhang T, O'Brien P, Boisvert JL, Price S, Hur W, Yang W, Deng X, Butler A, Choi HG, Chang JW, Baselga J, Stamenkovic I, Engelman JA, Sharma SV, Delattre O, Saez-Rodriguez J, Gray NS, Settleman J, Futreal PA, Haber DA, Stratton MR, Ramaswamy S, Mcdermott U, Benes CH (2012) Systematic identification of genomic markers of drug sensitivity in cancer cells. Nature 483: 570-575.

Grinberg I, Reis A, Ohana A, Taizi M, Cipok M, Tavor S, Rund D, Deutsch VR, Goldstein RS (2009) Engraftment of human blood malignancies to the turkey embryo: a robust new in vivo model. Leuk Res 33: 1417-1426.

Hagedorn M, Javerzat S, Gilges D, Meyre A, De Lafarge B, Eichmann A, Bikfalvi A (2005) Accessing key steps of human tumor progression in vivo by using an avian embryo model. Proc Natl Acad Sci USA 102: 1643-1648.

Kisselbach L, Merges M, Bossie A, Boyd A (2009) CD90 Expression on human primary cells and elimination of contaminating fibroblasts from cell cultures. Cytotechnology 59: 31-44.

Lynch TJ, Bell DW, Sordella R, Gurubhagavatula S, Okimoto RA, Brannigan BW, Harris PL, Haserlat SM, Supko JG, Haluska FG, Louis DN, Christiani DC, Settleman J, Haber DA (2004) Activating mutations in the epidermal growth factor receptor underlying responsiveness of non-small-cell lung cancer to gefitinib. N Engl J Med 350: 2129-2139.

Neesse A, Michl P, Frese KK, Feig C, Cook N, Jacobetz MA, Lolkema MP, Buchholz M, Olive KP, Gress TM, Tuveson DA (2011) Stromal biology and therapy in pancreatic cancer. Gut 60: 861-868.
Perez-Mancera PA, Guerra C, Barbacid M, Tuveson DA (2012) What we have learned about pancreatic cancer from mouse models. Gastroenterology 142(5): 1079-1092.

Polyak K, Weinberg RA (2009) Transitions between epithelial and mesenchymal states: acquisition of malignant and stem cell traits. Nat Rev Cancer 9: 265-273.

Rubio-Viqueira B, Jimeno A, Cusatis G, Zhang X, Iacobuzio-Donahue C, Karikari C, Shi C, Danenberg K, Danenberg PV, Kuramochi H, Tanaka K, Singh S, Salimi-Moosavi H, Bouraoud N, Amador ML, Altiok S, Kulesza P, Yeo C, Messersmith W, Eshleman J, Hruban RH, Maitra A, Hidalgo M (2006) An in vivo platform for translational drug development in pancreatic cancer. Clin Cancer Res 12: 4652-4661.

Sharma SV, Haber DA, Settleman J (2010) Cell line-based platforms to evaluate the therapeutic efficacy of candidate anticancer agents. Nat Rev Cancer 10: 241-253.

Shepherd TG, Theriault BL, Campbell EJ, Nachtigal MW (2006) Primary culture of ovarian surface epithelial cells and ascites-derived ovarian cancer cells from patients. Nat Protoc 1: 2643-2649.

Slamon DJ, Leyland-Jones B, Shak S, Fuchs H, Paton V, Bajamonde A, Fleming T, Eiermann W, Wolter J, Pegram M, Baselga J, Norton L (2001) Use of chemotherapy plus a monoclonal antibody against HER2 for metastatic breast cancer that overexpresses HER2. N Engl J Med 344: 783-792.

This work is published under the standard license to publish agreement. After 12 months the work will become freely available and the license terms will switch to a Creative Commons AttributionNonCommercial-Share Alike 3.0 Unported License.

Supplementary Information accompanies this paper on British Journal of Cancer website (http://www.nature.com/bjc) 